\title{
Discrete Viscous Threads
}
Miklós Bergou
Basile Audoly
Columbia University
UPMC Univ. Paris 06 \& CNRS
Etienne Vouga
Max Wardetzky
Eitan Grinspun
Columbia University
Universität Göttingen
Columbia University
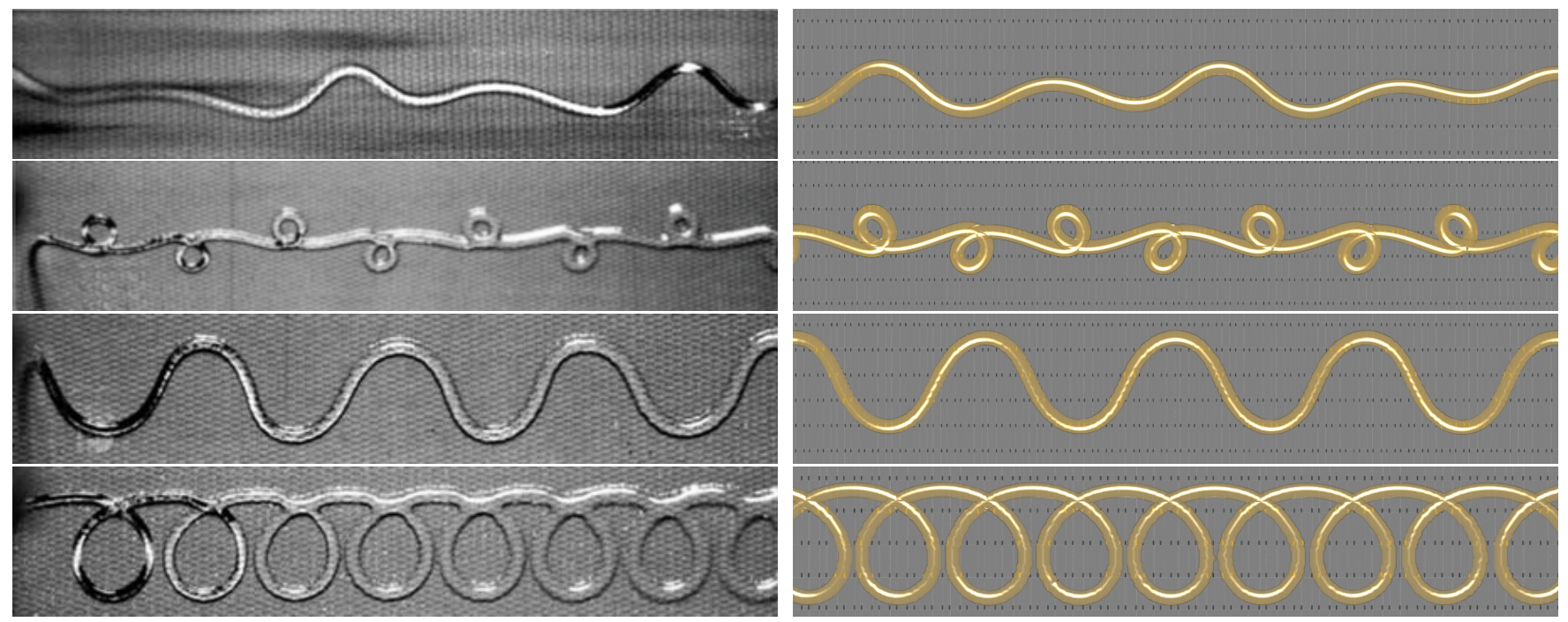

Figure 1: A thin thread of viscous fluid is poured onto a moving belt, creating a dazzling array of intricate patterns. Simulations using our model reproduce this rich and complex behavior. Translucent thread: experiment [Chiu-Webster and Lister 2006]; gold thread: simulation.

\section{Abstract}

We present a continuum-based discrete model for thin threads of viscous fluid by drawing upon the Rayleigh analogy to elastic rods, demonstrating canonical coiling, folding, and breakup in dynamic simulations. Our derivation emphasizes space-time symmetry, which sheds light on the role of time-parallel transport in eliminating - without approximation-all but an $O(n)$ band of entries of the physical system's energy Hessian. The result is a fast, unified, implicit treatment of viscous threads and elastic rods that closely reproduces a variety of fascinating physical phenomena, including hysteretic transitions between coiling regimes, competition between surface tension and gravity, and the first numerical fluidmechanical sewing machine. The novel implicit treatment also yields an order of magnitude speedup in our elastic rod dynamics.

CR Categories: I.3.7 [Computer Graphics]: Three-Dimensional Graphics and Realism-Animation

Keywords: viscous threads, coiling, Rayleigh analogy, elastic rods, hair simulation

\section{Introduction}

A curious little mystery of afternoon tea is the folding, coiling, and meandering of a thin thread of honey as it falls upon a freshly baked scone. Understanding the motion of this viscous thread is a gateway to simulation tools whose utility spans film-making, gaming, and engineering; for example, in over $30 \%$ of worldwide textile manufacturing processes, threads of viscous liquid polymers (often incorporating recycled materials) are entangled to form nonwoven fabric used in baby diapers, bandages, envelopes, upholstery, air ("HEPA") filters, surgical gowns, high-traffic carpets, erosion control, felt, frost protection, and tea sachets [Andreassen et al. 1997].

Viscous threads display fascinating behaviors that are challenging to accurately reproduce with existing simulation techniques. For example, a viscous thread steadily poured onto a moving belt creates a sequence of "sewing machine" patterns (see Fig. 1). While in theory, it is possible to accurately compute the motion of a viscous thread using a general, volumetric fluid simulator, there are no reports of successes to date, perhaps because the resolution needed for a sufficiently accurate reproduction requires prohibitively expensive runtimes.

In contrast to volumetric approaches, we model viscous threads by their formal analogy to elastic rods, for which relatively inexpensive computational tools are readily available. Both viscous threads and elastic rods are amenable to a reduced coordinate model operating on a centerline curve decorated with a cross-sectional material frame. Predicting the motion of viscous threads requires taking into account the competition between external forces, surface tension, and the material's resistance to stretching, bending, and twisting rates. Thus, with the exception of surface tension, which generally plays a negligible role for elastic materials, an existing implementation of stretching, bending, and twisting for an elastic rod can be easily repurposed for simulating a viscous thread. 

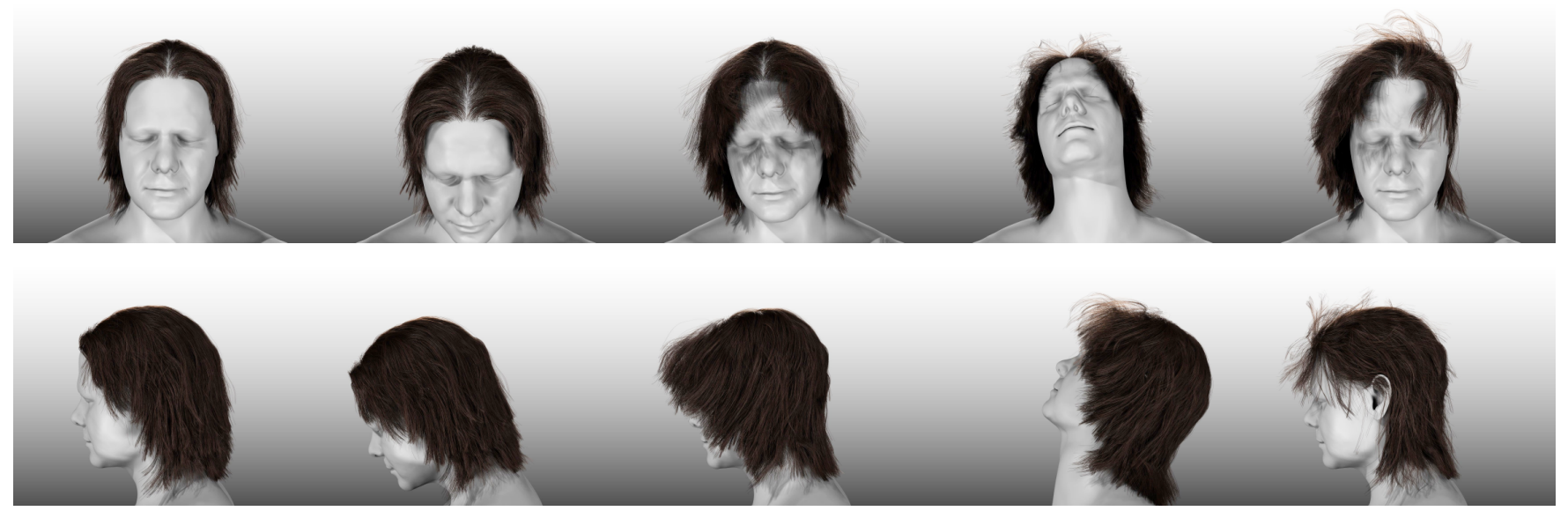

Figure 2: Using a time-parallel reference frame enables our implicit solver to operate on a banded linear system, yielding an order of magnitude speedup in hair simulation over the previous space-parallel formulation [Bergou et al. 2008]. Images courtesy of Weta Digital.

We use the Discrete Elastic Rods model [Bergou et al. 2008] as a starting point, which is validated against analytic and experimental data and adopted by academia [Chentanez et al. 2009] and industry [DiVerdi et al. 2010]. As originally formulated, Discrete Elastic Rods have a dense energy Hessian. We introduce the use of timeparallel reference frames in the representation of adapted framed curves, leading to locally supported energy stencils and a banded Hessian. Our derivation shows that the new model is formally equivalent to the original model while offering an order of magnitude improvement in computation of dynamics (see Fig. 2). Additionally, we observe that if the elastic setting might benefit from an implicit treatment, this benefit is doubly true for the viscous case, whose numerical stiffness makes explicit time integration problematic [Hauth et al. 2003]. We validate our discrete model for viscous threads against an as-yet unmet experimental benchmark, demonstrating for the first time the simulation of naturally occurring, periodic sewing machine patterns in poured viscous fluids.

\section{Related Work}

The graphical simulation of viscoelastic materials, including fluids and solids, encompasses hundreds of works covered in recent surveys [Witkin and Baraff 2001; Nealen et al. 2006; Bridson and Müller-Fischer 2007]. We briefly highlight lines of work and their relation to our coordinate-reduced Lagrangian representation of viscous threads.

Lagrangian approaches, such as ours, are adept at tracking a moving material occupying a small fraction of space. While we specifically focus on thin threads, others simulate more bulky viscoelastica using a variety of representations, such as points [Müller et al. 2004; Gerszewski et al. 2009], particles [Miller and Pearce 1989; Terzopoulos et al. 1991; Steele et al. 2004; Clavet et al. 2005], smoothed particle hydrodynamics [Desbrun and Gascuel 1996; Stora et al. 1999; Müller et al. 2003; Rafiee et al. 2007; Chang et al. 2009], and meshes [Terzopoulos and Fleischer 1988; O'Brien et al. 2002; Bargteil et al. 2007]. Wojtan et al. [2008] consider thin-featured viscous and plastic materials, embedding a high resolution surface in a coarse tetrahedral mesh for fast, physically plausible motion.

Lagrangian methods are also ideal for simulating elastic rods [Pai 2002; Bertails et al. 2006; Spillmann and Teschner 2007; Theetten et al. 2008]. In the finite element community, Boyer and Primault [2004] also discuss reference frames for adapted framed curves but avoid parametrizing the configuration by advocating the use of a Lie group integrator.

Eulerian treatments ensure uniform spatial sampling of large volumes, such as those needed for smoke [Foster and Metaxas 1997; Stam 1999; Fedkiw et al. 2001]. For liquids, the free surface must be tracked [Foster and Fedkiw 2001]. Batty and Bridson [2008] propose an elegant variational principle to enforce the free surface boundary condition, computing plausible motion for even the largest volumes of thick fluid that coil and fold; by contrast, our Lagrangian method accurately reproduces the coiling of even the thinnest 3D fluid threads. In Eulerian methods, viscoelastic materials require accumulated strain advection and additional elastic force terms [Goktekin et al. 2004; Irving 2007]. Thin features require special attention: Losasso et al. [2004] employ an octree data structure to capture small-scale visual detail. Hong and Kim [2005] consider the breakup of a liquid sheet and spurting of a liquid jet. Kim et al. [2009] extend the vortex sheet method to enhance fine details such as wiggling fluid sheets.

In the mechanics literature, the closest Eulerian approach is that of Oishi et al. [2008], who use a finite difference scheme based on an updated marker-and-cell method to study the buckling of a viscous jet based on the numerical solution of the 3D Navier-Stokes equations with free boundaries. Bonito et al. [2006] show results pertaining to $3 \mathrm{D}$ jet buckling based on an approach that combines finite elements with the method of characteristics.

Derivation of the viscous thread model begins with Trouton [1906], who identifies the effective modulus of a viscous string in tension. Entov and Yarin [1984] derive the full viscous thread model for 3D viscous flows, adding the effect of twist and curvature; the model is further explored in subsequent decades [Dewynne et al. 1992].

Numerical solution of 3D fluid jets is, to our knowledge, considered only once for general configurations, in the context of interactive painting [Lee et al. 2006]. This system produces impressive Jackson Pollock-style paintings using a heuristic coupling of the large-scale motion of the centerline to the equations of Eggers [1994] describing the flow inside the jet. By contrast, our model is based on the reduced coordinate formulation, and we do not need to solve numerically for the flow inside the thread.

While we pursue the general case, others develop numerics specialized to specific hypotheses: Skorobogatiy and Mahadevan [2000] consider the 2D, time-dependent problem of folding, where twist is 
identically zero. Ribe [2004] solves the non-linear boundary value problem for viscous coiling in the corotating frame, where the shape becomes stationary. Panda et al. [2008] present dynamic simulations of stretched viscous filaments in a geometry where bending and twist can be neglected.

Coiling is a canonical problem in the study of viscous threads that remains poorly understood. Taylor [1968] qualitatively explains the coils by analogy with the buckling of an elastic column in compression. Ribe [2006] presents a detailed comparison of simulations of steady coiling to experiments. Chiu-Webster and Lister [2006] have unveiled a variety of new experimental patterns in the dynamic regime that have remained, until now, numerically unreproduced.

\section{Adapted Framed Curves}

The geometry of an elastic rod or viscous thread is conveniently described by an adapted framed curve. Although this insight is not new [Kirchhoff 1859], we are about to see that not all representations for adapted framed curves are created equal when it comes to numerical performance and ease of implementation. In $\S 3.2$, we single out space-parallel and time-parallel reference frames for parametrizing an adapted framed curve. Whereas the former is explored by Bergou et al. [2008], the computational advantages of the latter have not previously been explored.

We present a development of the smooth setting alongside the discrete setting. As it turns out, the discrete picture not only spells out the actual implementation, but it often simplifies and provides intuition for the corresponding smooth derivation.

Smooth adapted framed curves For dynamics, we must consider the time evolution of the adapted framed curve. A timeevolving adapted framed curve consists of the centerline and material frame

$$
\mathbf{x}(s, t) \in \mathbb{R}^{3} \quad \text { and } \quad\left[\mathbf{d}_{1}(s, t) \quad \mathbf{d}_{2}(s, t) \quad \mathbf{d}_{3}(s, t)\right] \in \mathrm{SO}(3)
$$

where $s$ is the material coordinate, $t$ is time, and $\mathbf{d}_{1}, \mathbf{d}_{2}, \mathbf{d}_{3}$ are the three orthonormal director vectors. The frame is adapted to the centerline, which disallows shearing of the material frame by requiring that $\mathbf{d}_{3}$ lie tangent to the centerline, $\mathbf{t}=\mathbf{x}^{\prime} /\left|\mathbf{x}^{\prime}\right|$ (where the prime indicates differentiation with respect to the $s$ coordinate). Thus, $\mathbf{d}_{1}$ and $\mathbf{d}_{2}$ span the plane normal to the centerline's tangent, called the cross-section, and are referred to as the material directors. We assume that $s$ is an arc-length material parametrization of the undeformed configuration of the adapted framed curve. We denote quantities associated to the undeformed configuration of the material with an overline, so that $\overline{\mathbf{x}}^{\prime}(s, t)$ is unit length but $\mathbf{x}^{\prime}(s, t)$ may not be unit length.

Discrete adapted framed curves The development of the discrete model mimics that of the smooth setting: we define discrete adapted framed curves and their attendant deformation measures. A discrete adapted framed curve is a centerline given by a set of $n+2$ vertices (lower indices) and a set of $n+1$ adapted, orthonormal frames attached to each edge (upper indices),

$$
\mathbf{x}_{i} \in \mathbb{R}^{3} \quad \text { and } \quad\left[\begin{array}{lll}
\mathbf{d}_{1}^{j} & \mathbf{d}_{2}^{j} & \mathbf{d}_{3}^{j}
\end{array}\right] \in S O(3)
$$

for $0 \leq i \leq n+1$ and $0 \leq j \leq n$. We define adapted in the discrete case to mean that $\mathbf{d}_{3}^{j}$ lies along the edge formed by the adjacent vertices, $\mathbf{e}^{j}=\mathbf{x}_{j+1}-\mathbf{x}_{j}$, and is thus given by $\mathbf{d}_{3}^{j} \equiv \mathbf{t}^{j}=\mathbf{e}^{j} /\left|\mathbf{e}^{j}\right|$.

Before we discuss the coordinates we use to represent an adapted frame curve, we first define the purely geometric, kinematic quantities needed for dynamic simulation. Once we have these definitions, we will choose coordinates in $\S 3.2$ that most simplify the computation of these quantities.

\subsection{Kinematics}

The velocities and strains associated to the adapted framed curve are closely related, since the former depends on how the centerline and material frame change over time $t$, while the latter depends on how they change along arclength $s$, or space.

Centerline In the smooth setting, the kinematics of the centerline is governed by its velocity, $\dot{\mathbf{x}}(s, t)=\partial \mathbf{x} / \partial t$, and deformation gradient, $\mathbf{x}^{\prime}(s, t)=\partial \mathbf{x} / \partial s$. The deformation gradient is used to define the relative axial strain, $\varepsilon=\left|\mathbf{x}^{\prime}\right| /\left|\overline{\mathbf{x}}^{\prime}\right|-1$. In the discrete setting, we keep track of the velocities of the vertices, $\dot{\mathbf{x}}_{i}$, and define the edge-based relative axial strain as $\varepsilon^{j}=\left|\mathbf{e}^{j}\right| /\left|\overline{\mathbf{e}}^{j}\right|-1$.

Smooth material frame kinematics The material frame, always orthonormal, in the smooth case evolves over time via ${ }^{1}$

$$
\dot{\mathbf{d}}_{\alpha}=\boldsymbol{\omega} \times \mathbf{d}_{\alpha}, \quad \text { where } \quad \boldsymbol{\omega}=\mathbf{t} \times \dot{\mathbf{t}}+\omega_{3} \mathbf{t} .
$$

The angular velocity, $\omega$, is decomposed into $\mathbf{t} \times \dot{\mathbf{t}}$, which is the component required to keep the frame adapted and is fully determined by the moving centerline, and the rotation rate $\omega_{3}=\dot{\mathbf{d}}_{1} \cdot \mathbf{d}_{2}$ about the tangent, which is specific to the material frame.

Compare the spatial evolution of the material frame, which satisfies

$$
\mathbf{d}_{\alpha}^{\prime}=\boldsymbol{\Omega} \times \mathbf{d}_{\alpha}, \quad \text { where } \boldsymbol{\Omega}=\mathbf{t} \times \mathbf{t}^{\prime}+m \mathbf{t},
$$

to the temporal evolution; observe the space-time symmetry. The Darboux vector, $\boldsymbol{\Omega}$, is decomposed into the curvature binormal, $\kappa \mathbf{b}=\mathbf{t} \times \mathbf{t}^{\prime}$, adapting the frame along centerline traversal, and the twist, $m=\mathbf{d}_{1}^{\prime} \cdot \mathbf{d}_{2}$, about the tangent. Following Bergou et al. [2008], we can write the curvature (normal) vector in material coordinates as

$$
\kappa=\left(\kappa_{1}, \kappa_{2}\right)^{T}=\left(\mathbf{t}^{\prime} \cdot \mathbf{d}_{1}, \mathbf{t}^{\prime} \cdot \mathbf{d}_{2}\right)^{T}=\left(\kappa \mathbf{b} \cdot \mathbf{d}_{2},-\kappa \mathbf{b} \cdot \mathbf{d}_{1}\right)^{T} .
$$

Parallel transport Describing the kinematics of the material frame in the discrete case requires a key concept: the parallel transport from a unit vector $\mathbf{r}_{1}$ to another unit vector $\mathbf{r}_{2}$ is the minimum rotation that aligns $\mathbf{r}_{1}$ with $\mathbf{r}_{2}$ and can be formally computed as

$$
\mathrm{P}_{\mathbf{r}_{1}}^{\mathbf{r}_{2}} \equiv \mathrm{R}\left(\mathbf{r}_{1} \times \mathbf{r}_{2} /\left|\mathbf{r}_{1} \times \mathbf{r}_{2}\right|, \angle\left(\mathbf{r}_{1}, \mathbf{r}_{2}\right)\right),
$$

where $\mathrm{R}(\mathbf{r}, \psi)$ is the rotation about the unit vector $\mathbf{r}$ by an angle $\psi$, and $\angle\left(\mathbf{r}_{1}, \mathbf{r}_{2}\right)$ is the angle between $\mathbf{r}_{1}$ and $\mathbf{r}_{2}$.

Discrete material frame kinematics In adapting the material frame, the discrete follows the smooth picture, now as a two-step process: consider the tangent at two consecutive instants in time, $\mathbf{t}^{j}\left(t_{k-1}\right)$ and $\mathbf{t}^{j}\left(t_{k}\right)$. We first parallel transport the material frame, which aligns the tangents, and we then rotate by the angle $\omega_{3}^{j}\left(t_{k}\right)$ about $\mathbf{t}^{j}\left(t_{k}\right)$ to align the material frames. Thus, the material frames satisfy

$$
\mathbf{d}_{\alpha}^{j}\left(t_{k}\right)=\mathrm{R}\left(\mathbf{t}^{j}\left(t_{k}\right), \omega_{3}^{j}\left(t_{k}\right)\right) \circ \mathrm{P}_{\mathbf{t}^{j}\left(t_{k-1}\right)}^{\mathbf{t}^{j}\left(t_{k}\right)} \cdot \mathbf{d}_{\alpha}^{j}\left(t_{k-1}\right)
$$

In perfect analogy to discrete temporal evolution, the frame associated to edge $i-1$ maps to the subsequent edge in space by a finite

\footnotetext{
${ }^{1}$ Bergou et al. [2008] use $\boldsymbol{\omega}$ to denote the curvature vector expressed in material coordinates, which we denote using $\kappa$ instead.
} 
rotation, which is fully determined by the centerline shape up to the scalar twist $m_{i}$,

$$
\mathbf{d}_{\alpha}^{i}\left(t_{k}\right)=\mathrm{R}\left(\mathbf{t}^{i}\left(t_{k}\right), m_{i}\left(t_{k}\right)\right) \circ \mathrm{P}_{\mathbf{t}^{i-1}\left(t_{k}\right)}^{\mathbf{t}^{i}\left(t_{k}\right)} \cdot \mathbf{d}_{\alpha}^{i-1}\left(t_{k}\right) .
$$

Instead of replacing the finite rotation by an infinitesimal one (i.e., a skew-symmetric matrix), as often done in linearized elasticity, this geometrically nonlinear approach guarantees that our model remains objective (i.e., invariant under rigid motions).

For the discrete material curvatures, we follow a similar procedure to Bergou et al. [2008] and define the discrete material curvature at an interior vertex $1 \leq i \leq n$ as

$$
\boldsymbol{\kappa}_{i}=\frac{1}{2} \sum_{j=i-1}^{i}\left((\kappa \mathbf{b})_{i} \cdot \mathbf{d}_{2}^{j},-(\kappa \mathbf{b})_{i} \cdot \mathbf{d}_{1}^{j}\right)^{T}
$$

where $(\kappa \mathbf{b})_{i}=\frac{2 \mathbf{t}^{i-1} \times \mathbf{t}^{i}}{1+\mathbf{t}^{i-1} \cdot \mathbf{t}^{i}}$ is the vertex-based curvature binormal.

\subsection{Choosing among curve and angle representations}

To specify the configuration of an adapted framed curve, some set of coordinates must be chosen. Many choices abound, from fully reduced coordinates for representing an inextensible centerline and unshearable material frame [Bertails et al. 2006] to maximal coordinates for representing an extensible centerline and shearable material frame [Grégoire and Schömer 2007]. A hybrid approach, discussed below, uses an explicit centerline representation and reduced coordinates for the adapted frames [Langer and Singer 1996; Theetten et al. 2007; Bergou et al. 2008], which allows for simpler handling of collisions and direct manipulation while sidestepping complications of enforcing the unshearability constraint.

Reference directors Thus far, we have been focusing on arbitrary directors, which, intuitively, we associate with the material's principal axes. However, in order to separate material properties from geometry, it is useful to introduce another set of directors, which we call reference directors, which represent adapted frames that only depend on the (spatial or temporal) evolution of the centerline. We decorate reference directors, and all quantities associated to the reference frame, with an underline, $\left(\underline{\mathbf{d}}_{1}(s, t), \underline{\mathbf{d}}_{2}(s, t)\right)$. Note that any other

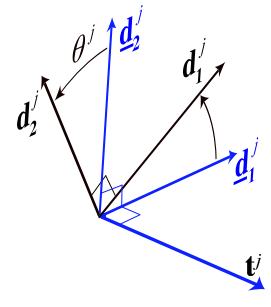
adapted frame, such as the material frame, can then be represented by an angle $\theta(s, t)$, which rotates (about the shared tangent) from the reference to the material frame. The discrete picture (see figure) assigns an angle $\theta^{j}$ to each edge:

$$
\begin{aligned}
& \mathbf{d}_{1}^{j}=\left(\cos \theta^{j}\right) \underline{\mathbf{d}}_{1}^{j}+\left(\sin \theta^{j}\right) \underline{\mathbf{d}}_{2}^{j} \\
& \mathbf{d}_{2}^{j}=-\left(\sin \theta^{j}\right) \underline{\mathbf{d}}_{1}^{j}+\left(\cos \theta^{j}\right) \underline{\mathbf{d}}_{2}^{j} .
\end{aligned}
$$

Using this representation, any combination of coordinates for the centerline $\mathbf{x}$ and the angles $\theta$ specifies a legal configuration for an adapted framed curve. The angular velocity and twist can be expressed in terms of these coordinates as

$$
\omega_{3}=\dot{\theta}+\underbrace{\dot{\mathbf{d}}_{1} \cdot \underline{\mathbf{d}}_{2}}_{\underline{\omega}_{3}} \quad \text { and } \quad m=\theta^{\prime}+\underbrace{\underline{\mathbf{d}}_{1}^{\prime} \cdot \underline{\mathbf{d}}_{2}}_{\underline{m}} .
$$

Here, $\underline{m}$ is the reference twist and $\underline{\omega}_{3}$ is the reference angular velocity, i.e., quantities associated to the reference frame. In the discrete setting, these quantities can be expressed as

$$
\omega_{3}^{j}\left(t_{k}\right)=\Delta \theta^{j}+\underline{\omega}_{3}^{j}\left(t_{k}\right) \text { and } m_{i}\left(t_{k}\right)=\Delta \theta_{i}+\underline{m}_{i}\left(t_{k}\right),
$$

where $\Delta \theta^{j}=\theta^{j}\left(t_{k}\right)-\theta^{j}\left(t_{k-1}\right), \Delta \theta_{i}=\theta^{i}\left(t_{k}\right)-\theta^{i-1}\left(t_{k}\right)$, and $\underline{\omega}_{3}^{j}\left(t_{k}\right)$ and $\underline{m}_{i}\left(t_{k}\right)$ are defined analogously to (1) and (2).

Not all reference directors are created equal How should we define the reference directors $\underline{\mathbf{d}}_{1}$ and $\underline{\mathbf{d}}_{2}$ ? In answering this, we (i) offer a novel unified treatment of spatial and temporal parallel transport and (ii) show that the newly introduced time-parallel frames offer a significant computational advantage over the previously considered space-parallel ones [Bergou et al. 2008].

We evaluate the choice of reference frames by favoring ease of computations required by dynamics simulations. In this setting, computational efficiency relies on minimizing the time needed to solve the equations of motion governed by the forces acting on the physical system. Computing the forces on the centerline requires the gradient of the strains defined in $\S 3.1$ with respect to vertex positions, and implicit integrators additionally require the Hessian of the strains. The bending and twisting strains contain reference-frame dependent quantities, so we must consider the variation of the reference directors, $\delta \underline{\mathbf{d}}_{\alpha}$, due to a variation in vertex positions, $\delta \mathbf{x}$, which is given in the continuous and discrete settings respectively by

$$
\int \frac{\partial \underline{\mathbf{d}}_{\alpha}(s, t)}{\partial \mathbf{x}(\sigma, t)} \cdot \delta \mathbf{x}(\sigma, t) \mathrm{d} \sigma \quad \text { and } \sum_{i=0}^{n+1} \frac{\partial \underline{\mathbf{d}}_{\alpha}^{j}\left(t_{k}\right)}{\partial \mathbf{x}_{i}\left(t_{k}\right)} \cdot \delta \mathbf{x}_{i}\left(t_{k}\right) .
$$

If the reference directors $\underline{\mathbf{d}}_{\alpha}^{j}\left(t_{k}\right)$ have nonlocal support (i.e., depend on vertices arbitrarily far away), the terms in this summation do not vanish, and the Hessian is dense. Thus, we seek to minimize this dependence and make the support as compact as possible.

Space-parallel frame Using the twist-free reference frame as proposed by Bergou et al. [2008] requires $\underline{m}=0$, which corresponds to parallel transporting the reference frame in space using $\underline{\mathbf{d}}_{\alpha}^{\prime}=\kappa \mathbf{b} \times \underline{\mathbf{d}}_{\alpha}$. Thus, given $\underline{\mathbf{d}}_{\alpha}(0, t)$ (the boundary conditions), an integration over the spatial dimension, $s$, defines $\underline{\mathbf{d}}_{\alpha}(s, t)$. This implies the reference directors $\underline{\mathbf{d}}_{\alpha}(s, t)$ depend on $\kappa \mathbf{b}(\sigma, t)$, and therefore on $\mathbf{x}(\sigma, t)$, for all $\sigma \leq s$.

In the discrete case, the twist-free frame requires $\underline{m}_{i}=0$ and is defined via

$$
\underline{\mathbf{d}}_{\alpha}^{i}\left(t_{k}\right)=\mathrm{P}_{\mathbf{t}^{i-1}\left(t_{k}\right)}^{\mathbf{t}^{i}\left(t_{k}\right)} \cdot \underline{\mathbf{d}}_{\alpha}^{i-1}\left(t_{k}\right)
$$

with the boundary conditions $\underline{\mathbf{d}}_{\alpha}^{0}\left(t_{k}\right)$. As a consequence, since the space-parallel frame $\underline{\mathbf{d}}_{\alpha}^{j}\left(t_{k}\right)$ is defined iteratively in terms of the tangents of all edges $\{0, \ldots, j\}$, the terms in the summation in (3) are nonzero for all $i \leq j+1$. Thus, to compute the force acting on vertex $i$, we must add up contributions from all edges $j \geq i-1$, which leads to a costly to compute dense energy Hessian.

Bergou et al. [2008] do not encounter this difficulty because they consider explicit time integration. They also note that for the special case of isotropic bending response, straight undeformed configuration, and quasistatic material frame, the space-parallel frame eliminates all reference-frame dependent quantities. We believe that indeed, for this special case there is no computationally more efficient solution. However, if an implicit time integrator is used and any of these specialized assumptions are lifted, the space-parallel reference frame becomes undesirable but fortunately avoidable.

Time-parallel frame The reference frame must stay adapted to the centerline - for any change in the centerline's tangent, the reference directors must follow (i.e., $\underline{\mathbf{d}}_{\alpha}(s, t)$ must at least depend on $\mathbf{t}(s, t))$. Thus, one natural choice is to always rotate the reference 


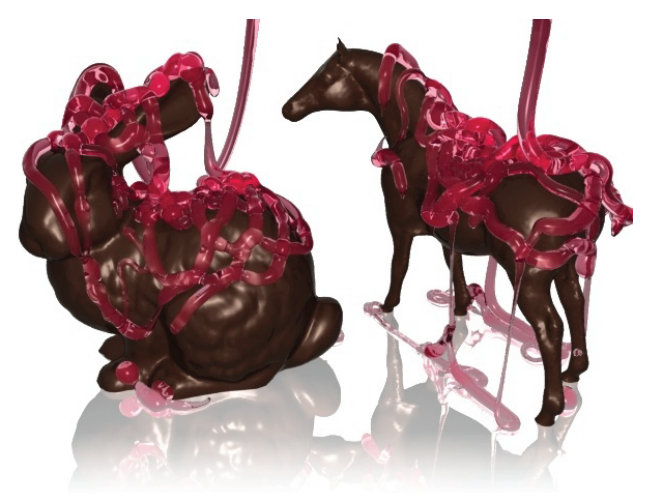

Figure 3: Discrete Viscous Threads offers a computationally efficient model for simulating scenes such as this.

frame by the minimum amount needed to keep it adapted. This selects the frame having zero tangential angular velocity, $\underline{\omega}_{3}=0$, corresponding to parallel transport in time using $\underline{\mathbf{d}}_{\alpha}=(\mathbf{t} \times \dot{\mathbf{t}}) \times \underline{\mathbf{d}}_{\alpha}$. Given the initial reference directors $\underline{\mathbf{d}}_{\alpha}(s, 0)$, we integrate over time to get the current reference directors $\underline{\mathbf{d}}_{\alpha}(s, t)$.

Analogously, for the discrete case, if $\underline{\omega}_{3}^{j}=0$, the discrete ODE

$$
\underline{\mathbf{d}}_{\alpha}^{j}\left(t_{k}\right)=\mathrm{P}_{\mathbf{t}^{j}\left(t_{k-1}\right)}^{\mathbf{t}^{j}\left(t_{k}\right)} \cdot \underline{\mathbf{d}}_{\alpha}^{j}\left(t_{k-1}\right)
$$

with initial conditions $\underline{\mathbf{d}}_{\alpha}^{j}\left(t_{0}\right)$ governs the frame's temporal evolution. During a simulation, this integration can be done incrementally - only the reference frame at the current time step must be known to advance it to the next time step. In fact:

Given the reference frame at time $t_{k-1}$, the time-parallel reference frame $\underline{\mathbf{d}}_{\alpha}^{j}\left(t_{k}\right)$ only depends on $\mathbf{t}^{j}\left(t_{k}\right)$, and the only nonzero terms in (3) are $i \in\{j, j+1\}$. Thus, the force stencil is local and the energy Hessian is banded.

In concurrent work, Kaldor et al. [2010] also propose the use of the time-parallel reference frame and present an efficient contact handling algorithm for simulating character-scale, yarn-based cloth. They call attention to the fact that the local support of the stencil allows one to easily parallelize the force computations, resulting in a computational advantage even for explicit time integration.

\section{Elastic Rods}

We briefly present background on elastic rods. In the discrete case, the elastic stretching, twisting, and bending potentials are given by

$$
\begin{aligned}
& E_{s}=\frac{1}{2} \sum_{j=0}^{n} k_{\mathrm{s}}^{j}\left(\varepsilon^{j}\right)^{2}\left|\overline{\mathbf{e}}^{j}\right|, \\
& E_{t}=\frac{1}{2} \sum_{i=1}^{n} \beta_{i} \frac{\left(m_{i}-\bar{m}_{i}\right)^{2}}{\bar{l}_{i}}, \\
& E_{b}=\frac{1}{2} \sum_{i=1}^{n} \frac{1}{\bar{l}_{i}}\left(\boldsymbol{\kappa}_{i}-\overline{\boldsymbol{\kappa}}_{i}\right)^{T} \mathrm{~B}_{i}\left(\boldsymbol{\kappa}_{i}-\overline{\boldsymbol{\kappa}}_{i}\right),
\end{aligned}
$$

where $\bar{l}_{i}=\frac{1}{2}\left(\left|\overline{\mathbf{e}}^{i-1}\right|+\left|\overline{\mathbf{e}}^{i}\right|\right)$ is the Voronoi length associated to a vertex. The metric $B$ measures anisotropic bending response along the material axes $\mathbf{d}_{1}$ and $\mathbf{d}_{2}$. The subtraction of $\overline{\boldsymbol{\kappa}}_{i}$ accommodates curved undeformed configurations.
Elastic force stiffnesses In order to obtain forces, we consider derivatives of the above elastic potentials with respect to the material coordinates. Physical force stiffnesses depend on the geometry of the rod's cross-section. In our model, we assume that each edge has an elliptical cross-section with major and minor radii given by $a^{j}$ and $b^{j}$, respectively, so that the cross-sectional area is $A^{j}=\pi a^{j} b^{j}$. At vertices, we define $a_{i}=\left(a^{i-1}+a^{i}\right) / 2$ (and likewise for $b_{i}$ ) so that the cross-sectional area satisfies $A_{i}=$ $\pi a_{i} b_{i}$. The stretching, twisting, and bending stiffness can be written as [Landau and Lifshitz 1981]

$$
k_{\mathrm{s}}^{j}=E A^{j}, \quad \beta_{i}=\frac{G A_{i}\left(a_{i}^{2}+b_{i}^{2}\right)}{4}, \quad \mathrm{~B}_{i}=\frac{E A_{i}}{4}\left(\begin{array}{cc}
a_{i}^{2} & 0 \\
0 & b_{i}^{2}
\end{array}\right),
$$

respectively. Here $E$ denotes Young's modulus and $G$ denotes the shear modulus of the material. Note that stretching stiffness is associated with edges, while bending and twisting stiffnesses are associated with vertices.

Explicit gradient and Hessian For efficiency of computation within the framework of an implicit time-stepping scheme, where the nonlinear Euler-Lagrange equation is solved (e.g., using Newton's method), we require explicit expressions for both the energy gradient and Hessian. While those are straightforward to obtain for the stretching part, the calculation of the energy gradient and Hessian for the twisting and bending parts turns out to be nontrivial. The final expressions are provided in Appendix A.

\section{Viscous Threads}

The general form of the elastic potentials defined in the previous section can be written in terms of a geometric measure of deformation $\epsilon$ (elongation, material curvature, or twist) as

$$
E_{\mathrm{el}}=\frac{1}{2} \int k_{\mathrm{el}}(\epsilon-\bar{\epsilon})^{2} \mathrm{~d} s .
$$

Elastic forces are then computed by taking variations of the elastic potential with respect to the material coordinates.

Our treatment of viscous threads is based on Rayleigh's observation that a viscous formulation derives from an elastic formulation when velocities replace positions and strain rates replace strains [Strutt 1945]. By analogy with the elastic case, the viscous model can be derived starting from the dissipative potential

$$
E_{\mathrm{vis}}=\frac{1}{2} \int k_{\mathrm{vis}} \dot{\epsilon}^{2} \mathrm{~d} s
$$

defined in terms of the rate of change of the geometric deformations. The viscous forces are then computed by taking variations of the dissipative potential with respect to the time derivatives (velocities) of the material coordinates. Compared to the elastic regime, this amounts to replacing internal elastic forces by internal viscous ones in Newton's equations of motion.

The fluid-as-thread paradigm The above model for viscous threads is valid under the assumption that the thickness is much smaller than any longitudinal length scale and that the curvature of the centerline is everywhere much smaller than the inverse of thickness. This implies that the thread can locally be well approximated by a cylinder. Computationally, the thread model offers tremendous advantages over a full-blown fluid treatment as it encapsulates all the small-scale details of the flow into effective parameters, thereby allowing the use of mesh sizes significantly larger than the thickness of the thread without loss of accuracy. 


\subsection{Discrete Setting}

Radovitzky and Ortiz [1999] followed by Kharevych et al. [2006] introduce a variational formulation for viscosity, writing viscous forces as the gradient of the discrete dissipative potential

$$
E_{\mathrm{vis}}=\frac{1}{2} \sum_{i}\left(k_{\mathrm{vis}}\right)_{i}\left(\frac{\epsilon_{i}\left(t_{k+1}\right)-\epsilon_{i}\left(t_{k}\right)}{h_{k}}\right)^{2},
$$

where the parenthesized expression is the discrete strain rate. Compared to the elastic regime, strains at time $t_{k}$ refer to the undeformed configuration, while strains at time $t_{k+1}$ refer to the deformed one. Considering variations with respect to material coordinates scaled by the inverse of the time step $h_{k}=t_{k+1}-t_{k}$, one obtains for the viscous force

$$
F_{\mathrm{vis}}=-\sum_{i} \frac{\left(k_{\mathrm{vis}}\right)_{i}}{h_{k}}\left(\epsilon_{i}\left(t_{k+1}\right)-\epsilon_{i}\left(t_{k}\right)\right) \frac{\partial \epsilon_{i}\left(t_{k+1}\right)}{\partial \mathbf{x}} .
$$

By folding the division by $h_{k}$ directly into the viscous stiffnesses (see below), this formulation of viscosity requires only a trivial modification to an elastic implementation: before each time step, we set the undeformed to the current strain, $\bar{\epsilon}_{i}=\epsilon_{i}\left(t_{k}\right)$ (see $\S 6$ ).

Viscous force stiffnesses In the case of viscous threads, we assume a cylindrical shape of the fluid along the edges, with radius given by $\left(a^{j}=b^{j}=r^{j}\right)$, and we set $r_{i}=\left(r^{i-1}+r^{i}\right) / 2$ at vertices. Thus, the cross-sectional area at edges and vertices is given by $A^{j}=\pi\left(r^{j}\right)^{2}$ and $A_{i}=\pi r_{i}^{2}$, respectively. Requiring incompressibility of the fluid, the stiffnesses then take the form [Ribe 2004]

$$
k_{\mathrm{s}}^{j}=\frac{3 \mu A^{j}}{h_{k}}, \quad \beta_{i}=\frac{\mu A_{i} r_{i}^{2}}{2 h_{k}}, \quad \mathrm{~B}_{i}=\frac{3 \mu A_{i} r_{i}^{2}}{4 h_{k}} \mathrm{I}_{2},
$$

where $\mu$ is the dynamic viscosity of the fluid, $h_{k}$ is the time step size, and $\mathrm{I}_{2}$ is the $2 \times 2$ identity matrix.

\subsection{Conservation of volume}

Throughout, we assume that the fluid is incompressible with uniform density, leading to the volume of the fluid to be conserved. In the discrete setting, we consider the volume to be an edge-based quantity given by $V^{j}=\pi\left(r^{j}\right)^{2}\left|\mathbf{e}^{j}\right|$, corresponding to the volume of the edge-based cylinder. We enforce volume conservation by updating $r^{j}$ at the end of each time step using $r^{j}=\sqrt{V^{j} /\left(\pi\left|\mathbf{e}^{j}\right|\right)}$, which makes the radius of the edge a function of the position of the two incident vertices. Note that since the stiffnesses $k_{\mathrm{s}}, \beta$, and $\mathrm{B}$ all depend on the radius, they must be updated, too.

\subsection{Surface tension}

Viscous threads are set apart from elastic rods because the effects of surface tension must be considered, reflecting the material's desire to minimize its overall surface area. The energy associated to surface tension is thus proportional to the surface area of the fluid, with constant of proportionality $\gamma$. In the discrete setting, we consider surface area (not to be confused with cross-sectional area) to be a vertex-based quantity, $\mathcal{A}_{i}=\pi\left(r^{i-1}+r^{i}\right) \sqrt{l_{i}^{2}+\left(r^{i}-r^{i-1}\right)^{2}}$, corresponding to the lateral surface area of a truncated cone with bottom and top given by the cross-sectional circles at edge midpoints. Using the relation between radius $r^{i}$ and volume $V^{i}$ from $\S 5.2$, we can express $\mathcal{A}_{i}$ in terms of $V^{i-1}, V^{i},\left|\mathbf{e}^{i-1}\right|$, and $\left|\mathbf{e}^{i}\right|$ only. Then $E_{\text {surf }}=\sum_{i=1}^{n} \gamma \mathcal{A}_{i}$ is the requisite energy associated to surface tension.

\subsection{Merging}

When two threads graze, surface tension attracts them, trying to minimize lateral boundary area, whereas viscosity resists their relative motion. While an exact account of merging would require a full 3D simulation, we derive a qualitatively correct treatment here.

Geometry. We consider the merging of two balls of identical radii $r=\left(r_{1}+r_{2}\right) / 2$ centered at points along the centerline of closest approach in the simulation, separated by a distance $d(t)$. We use $r_{1}$ and $r_{2}$ to denote the radii of the current points of closest approach. Let $\mathcal{V}(d)$ be the union of the two spheres, $\partial \mathcal{V}(d)$ its boundary (two portions of spheres), $\mathcal{A}(d)$

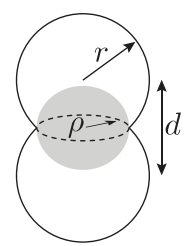
the boundary's area, and $\rho=\sqrt{r^{2}-(d / 2)^{2}}$ the radius of of the intersection region, as depicted in the incident figure.

Physics. The contribution of surface tension to the merging force derives from the potential energy $\gamma \mathcal{A}(d)$; this yields a force $\gamma\left(-\mathcal{A}^{\prime}(d)\right)$. The second factor is purely geometrical and can be calculated as $\left(-\mathcal{A}^{\prime}(d)\right)=2 \pi r$, which happens not to depend on $d$. On the other hand, the viscous flow involves a typical velocity $\dot{d}$ in a region whose volume is comparable to that of a sphere of radius $\rho$, shown in light gray in the picture. The power dissipated by this viscous flow can be estimated as $\mu \frac{4 \pi}{3} \rho^{3}(\dot{d} / \rho)^{2}$ and by division with respect to $\dot{d}$ we obtain the viscous contribution to the merging force. Summing up the two contributions, we obtain the attractive merging force $F_{\mathrm{m}}=2 \pi \gamma r-\mu \frac{4 \pi}{3} \rho|\dot{d}|$ acting along the direction of the points of closest approach.

\subsection{Adaptivity}

Viscous threads tend to stretch significantly, necessitating remeshing to avoid poor sampling (refer to Spillmann and Teschner [2008] for adaptation of elastic rods). We subdivide any edge $j$ whose length exceeds a prescribed discretization threshold into two edges and insert a new midpoint vertex. The position of the vertex is chosen by interpolating the position of the four vertices $\mathbf{x}_{j-1}, \mathbf{x}_{j}, \mathbf{x}_{j+1}, \mathbf{x}_{j+2}$ using a cubic Lagrange polynomial, and the velocity of the vertex is chosen to conserve the centerline's momentum. The newly created edges are assigned new radii, with the ratio of the radii chosen by interpolating the radii of the three edges $r^{j-1}, r^{j}, r^{j+1}$ using a quadratic Lagrange polynomial and absolute value chosen to conserve total volume.

In addition to remeshing to avoid excessive stretching, we consider topological changes caused by the breakup of the thread. We model this by removing any edge whose radius falls below a threshold.

\section{Simulation loop}

The algorithm for rods and threads is identical, with four exceptions: updating undeformed strains and stiffnesses, accounting for surface tension and merging, conserving volume, and remeshing.

Letting $\mathbf{q}=\left(\mathbf{x}_{0}, \theta^{0}, \cdots, \mathbf{x}_{n}, \theta^{n}, \mathbf{x}_{n+1}\right)^{T}$ and $h_{k}=t_{k+1}-t_{k}$, we apply Euler integration (implicit on internal and explicit on external forces), using Newton's method to solve

$$
\begin{aligned}
\mathrm{M} \Delta \dot{\mathbf{q}}-h_{k} \mathbf{F}_{\text {int }}\left(\mathbf{q}\left(t_{k}\right)+\Delta \mathbf{q}\right) & =h_{k} \mathbf{F}_{\text {ext }}\left(t_{k}, \mathbf{q}\left(t_{k}\right), \dot{\mathbf{q}}\left(t_{k}\right)\right) \\
\Delta \mathbf{q}-h_{k} \Delta \dot{\mathbf{q}} & =h_{k} \dot{\mathbf{q}}\left(t_{k}\right)
\end{aligned}
$$

for the increments to positions, $\Delta \mathbf{q}=\mathbf{q}\left(t_{k+1}\right)-\mathbf{q}\left(t_{k}\right)$, and velocities, $\Delta \dot{\mathbf{q}}=\dot{\mathbf{q}}\left(t_{k+1}\right)-\dot{\mathbf{q}}\left(t_{k}\right)$. The lumped mass matrix M assigns 
each vertex the mass $m_{i}=\rho\left(V^{i-1}+V^{i}\right) / 2$ of its Voronoi cell, where $\rho$ is the (volumetric) density of the material and $V^{i-1}$ and $V^{i}$ are the volumes of the incident edges. The implicit treatment also stably allows accounting for cross-sectional inertia, assigning each $\theta^{j}$ the inertia $I^{j}=\rho V^{j}\left(r^{j}\right)^{2} / 2$. In the

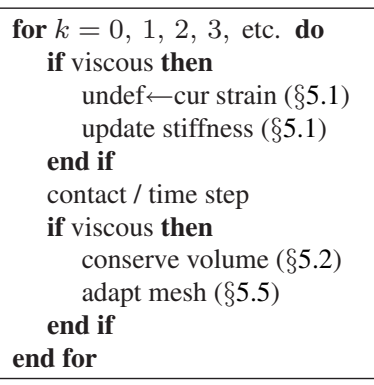
thin limit, where cross-sectional inertia vanishes, we may assign the $\left\{\theta^{j}\right\}$ variables zero inertia to maintain the material frames in quasistatic equilibrium [Bergou et al. 2008]. As theoretically predicted, we do not discern significant differences.

Newton update Each iteration of Newton's method provides a new guess for $\left\{\mathbf{x}_{i}\left(t_{k+1}\right)\right\}$ and $\left\{\theta^{j}\left(t_{k+1}\right)\right\}$. The material frame at the current iteration is given by parallel transport of the reference frame from the start of step to the current iterate followed by a rotation by $\theta^{j}$. The computation of forces at the current iteration must account for this parallel transport; if the reference frame is already adapted to the current iterate, parallel transport is the identity operation, and only variations away from the identity must be considered, with correspondingly simpler force expressions. We take advantage of this observation by changing to convenient coordinates: at every iteration, we update the reference frame so it is adapted to the current iterate. This change of coordinates requires updating $\left\{\theta^{j}\left(t_{k}\right), \dot{\theta}^{j}\left(t_{k}\right), \dot{\theta}^{j}\left(t_{k+1}\right)\right\}$, and quantities dependent on these. In addition, the reference frame accumulates twist at each iteration, which is accounted for by parallel transporting $\underline{\mathbf{d}}_{1}^{i-1}$ from $\mathbf{t}^{i-1}$ to $\mathbf{t}^{i}$, rotating about $\mathbf{t}^{i}$ by angle $\underline{m}_{i}$, and measuring the angle $\Delta \underline{m}_{i}$ between the result and $\underline{\mathbf{d}}_{1}^{i}$, corresponding to the change in twist: $\Delta \underline{m}_{i}=\angle\left(\mathrm{R}\left(\mathbf{t}^{i}, \underline{m}_{i}\right) \circ \mathrm{P}_{\mathbf{t}^{i-1}}^{\mathrm{t}^{i}} \cdot \underline{\mathbf{d}}_{1}^{i-1}, \underline{\mathbf{d}}_{1}^{i}\right)$.

\section{Results}
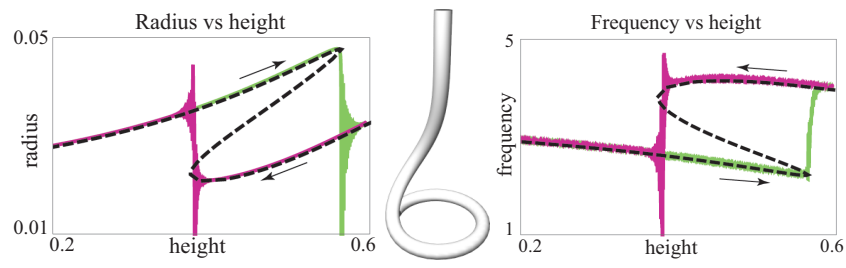

Figure 4: A viscous thread falling onto a table coils (middle) with the radius and frequency determined by the drop height. We compare computed stable radius (left) and coiling frequency (right) to that predicted by theory from Ribe [2004] (dashed lines) for different drop heights, first by slowly increasing the simulated height (green line) and then by decreasing (purple line).

Viscous coiling When honey pours from a bottle, the tail of the thread rapidly coils as it hits a table. The radius and frequency of the coil formation depend on the height of the bottle (see Fig. 4). Our simulation agrees with results predicted theoretically and validated experimentally by Ribe [2004]. In addition, our simulation shows a hysteretic effect also seen in real-world experiments [Ribe et al. 2006]: as the height of the container is slowly changed by first moving it upward and then back down, the simulation shows different stable coiling modes for the same heights. Physical parameters: volumetric density $\rho=5 \times 10^{-4}$, viscosity $\mu=0.2$, gravity $g=9.81$, surface tension $\gamma=0$, diameter of the thread at the container $d=0.09$, speed of thread as it leaves container $U_{0}=0.615$. Plot units: nondimensional units measured with respect to $H^{*}=\left(\mu /\left(\rho^{2} g\right)\right)^{1 / 3}$ for length and $\Omega^{*}=\left(g^{2} \rho / \mu\right)^{1 / 3}$ for frequency.

Fluid-mechanical sewing machine Replacing the steady table by a moving conveyor belt yields a striking array of patterns and, as the belt speed or drop height is changed, transitions between coiling and meandering regimes. For sufficiently high belt speed, the thread forms a catenary, whose shape is governed mainly by the interaction of gravity, axial viscosity, and surface tension.

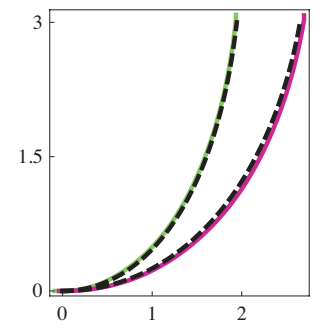

The incident figure shows the analytical result, derived by ChiuWebster and Lister [2006] as a dashed black line both with (right) and without (left) surface tension. Our simulation accurately reproduces both cases (green corresponds to no surface tension and purple to nonzero surface tension). Physical parameters: drop height: $3 \mathrm{~cm}$, belt speed: $0.7 \mathrm{~cm} / \mathrm{s}$, density $\rho=0.996 \mathrm{~g} / \mathrm{cm}^{3}$, viscosity $\mu=$ $273.6 \mathrm{~g} / \mathrm{cm} \cdot \mathrm{s}$, surface tension $\gamma=10 \mathrm{~g} / \mathrm{s}^{2}$, gravity $\mathrm{g}=100 \mathrm{~cm} / \mathrm{s}^{2}$, diameter at nozzle $d=0.8 \mathrm{~cm}$, speed of flow at nozzle $U_{0}=0.06 \mathrm{~cm} / \mathrm{s}$.

As the belt speed is lowered, the thread's tail draws distinct patterns on the belt (see Fig. 1). Our simulation reproduces many of the observed patterns, which have never been numerically reproduced previously. Fluid parameters: see [Morris et al. 2008].

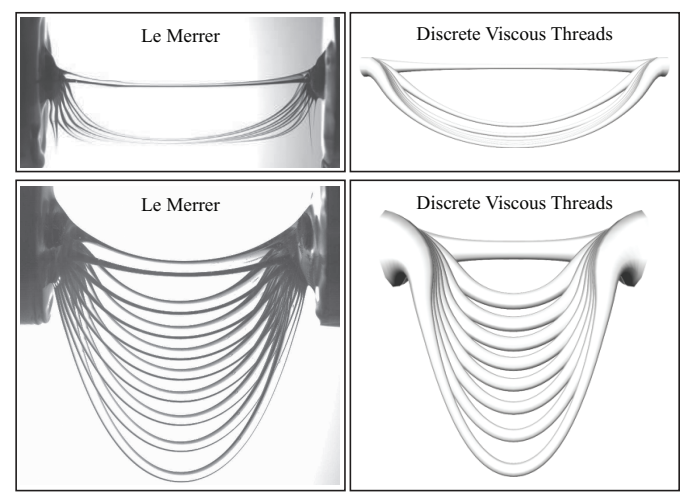

Figure 5: As a viscous filament held at two ends sags under gravity, the balance between the effects of gravity and surface tension leads to either a " $U$ " shape (top) or catenary (bottom).

Competition of surface tension and gravity When a viscous filament is suspended horizontally between two plates and allowed to sag under gravity, it tends to evolve into one of two shapes: (i) a catenary, which is characterized by near-uniform strain along the centerline at each point in time and a steady decrease in the height of the middle of the thread; or (ii) a "U" shape, where the center of the thread falls a small distance and then stops. In the latter case, surface tension drains fluid away from the center and towards the ends, increasing the strain at the thread's center until it snaps. Our simulation agrees with experiments of Le Merrer et al. [2008] in qualitative behavior (see Fig. 5), trajectory of the centerline's midpoint, and phase diagram (see Fig. 6). Physical parameters: oil density $0.907 \mathrm{~g} / \mathrm{cm}^{3}$, viscosity $100 \mathrm{~g} / \mathrm{cm} \cdot \mathrm{s}$, surface tension $20 \mathrm{~g} / \mathrm{s}^{2}$, plates $2.5 \mathrm{~cm}$ apart, thread diameter at centerline midpoint $0.175 \mathrm{~cm}$ for catenary and $0.033 \mathrm{~cm}$ for $\mathrm{U}$. 
Figure 6: A thread forms either a catenary (green circle) or U (purple square) as it falls, depending on its length $L$ and diameter $D$. The boundary fits the law $L D=4.7 a^{2}$, where $a$ is the capillary length (determined by fitting to the data), in accordance with theory of Le Merrer et al. [2008].

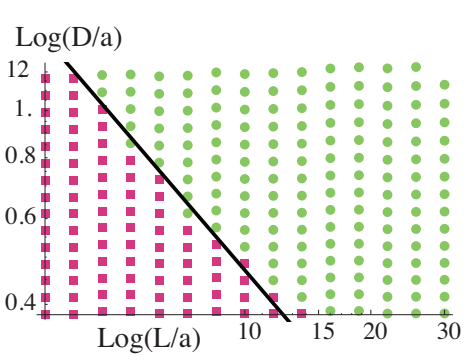

Implicit versus explicit Viscosity (damping) results in stiff equations of motion [Hauth et al. 2003], in turn typically imposing stringent limits on the stable time step. Indeed, for our simulations of viscous materials, we observe severe instabilities under explicit integration. Our implicit implementation allows for four orders of magnitude larger time steps than our corresponding explicit implementation. Despite the need to solve one or more linear systems each time step, the vastly fewer number of required steps translates into three orders of magnitude faster runtimes (see Fig. 3 and Table 1). The balance in favor of an implicit approach is significantly boosted by the structure of the time-parallel formulation's Hessian, which is symmetric and banded. This tames both storage and computational complexity: the number of nonzero entries that must be computed and stored grows linearly with the number of vertices, and the linear system required for time stepping can be solved in $O(n)$ time using standard routines from LAPACK.

\begin{tabular}{c|c|c|c|c|c|}
\multicolumn{1}{c}{ Figure } & \multicolumn{1}{c}{ Vertices } & \multicolumn{1}{c}{ Time step } & \multicolumn{1}{c}{ Fastest frame } & \multicolumn{1}{c}{ Slowest frame } & Average frame \\
\cline { 2 - 6 } 1 & 180 & $10 \mathrm{~ms}$ & $0.241 \mathrm{~ms}$ & $59.7 \mathrm{~ms}$ & $16.8 \mathrm{~ms}$ \\
\cline { 2 - 6 } 2 & 54460 & $10 \mathrm{~ms}$ & $23.5 \mathrm{~s}$ & $3 \mathrm{~m} 46 \mathrm{~s}$ & $1 \mathrm{~m} 17 \mathrm{~s}$ \\
\hline 3 & $200-1500$ & $0.208 \mathrm{~ms}$ & $0.292 \mathrm{~ms}$ & $0.916 \mathrm{~s}$ & $0.202 \mathrm{~s}$ \\
\hline 4 & $40-350$ & $40 \mathrm{~ms}$ & $0.05 \mathrm{~ms}$ & $35.1 \mathrm{~ms}$ & $3.64 \mathrm{~ms}$ \\
\hline 5 & 51 & $1 \mathrm{~ms}$ & $3.84 \mathrm{~ms}$ & $56.9 \mathrm{~ms}$ & $25.5 \mathrm{~ms}$ \\
\hline
\end{tabular}

Table 1: This table lists the computational cost for examples representative of the results depicted in the figures. Timings for the hair simulation (Fig. 2) were measured on a multi-threaded application running on a 6 core machine running at $3.07 \mathrm{GHz}$. The rest of the timings were measured on a single-threaded application running on a $3.166 \mathrm{GHz}$ machine.

Hair dynamics The implicit solver afforded by the time-parallel formulation results in a multi-fold performance benefit over our explicit implementation even for simulating elastic rods. For a 300 frame simulation of an elastic rod with 200 vertices, the total time spent in computing the dynamics of the rod (without collisions) was 0.488 seconds using our implicit code and 4.22 seconds using our explicit code. Using the implicit code, the stable time step was $70 \times$ larger, which allowed for far fewer sub-steps per frame. From a production standpoint, such as for creating scenes like that depicted in Fig. 2 and the accompanying video, the implicit formulation offers two advantages over existing production-quality alternatives: it is based on a continuum model, which greatly aids in setting up material parameters and consistent visual styles, and it is stable, allowing the user to focus on how the simulation looks without simultaneously adjusting discretization parameters to maintain stability.

Limitations and future work While we have been able to successfully validate our model against a wide range of theoretical as

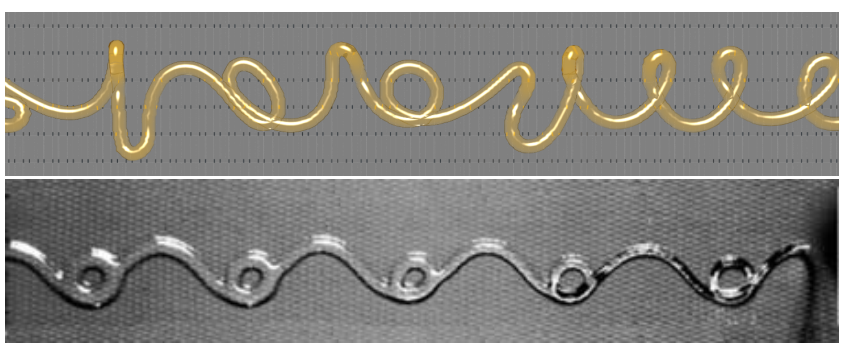

Figure 7: Some of the patterns for the sewing machine were observed only transiently, such as the " $W$ " pattern shown here.

well as experimental results concerning viscous threads, there are limitations imposed by our assumptions. We assume that the fluid is Newtonian and that its configuration is well represented using an adapted framed curve. When these assumptions are not valid, as happens for instance if the jet becomes very thick, the formulation presented here is no longer expected to produce valid results.

We additionally assume that the cross section of the thread remains circular (unlike for elastic rods) because of the effect of surface tension, so we neglect any motion of the fluid that would act to change the cross section. This assumption can be violated, for example, when the thread is laid down on a table and flattens out over time due to contact and gravity. In more general terms, we consider the simulation of anisotropic viscous threads or viscous sheets an interesting direction for future work.

In the fluid-mechanical sewing machine experiment, some patterns were observed transiently (see Fig. 7); for some belt speeds and nozzle heights, we observed a superposition of patterns and the attendant beat frequency. It is not yet fully understood, both in experiment and in numerics, how the system chooses one specific pattern when several of them are in competition. We hope that numerical tools such as those developed here can help us to gain an understanding of these phenomena.

\section{Acknowledgments}

We thank Alasdair Coull of Weta Digital for producing the hair simulation depicted in Fig. 2 and Sebastian Sylwan and Joe Letteri, both of Weta Digital, for facilitating the collaborative technology transfer process. This research is supported in part by the Sloan Foundation, the NSF (CAREER Award CCF-06-43268 and grants IIS-09-16129, IIS-05-28402, CNS-06-14770), and generous gifts from Adobe, ATI, Autodesk, mental images, NVIDIA, Side Effects Software, the Walt Disney Company, and Weta Digital.

\section{References}

Andreassen, E., Gundersen, E., Hinrichsen, E. L., And Langtangen, H. P. 1997. Numerical Methods and Software Tools in Industrial Mathematics. Birkhäueser, Boston, ch. A mathematical model for the melt spinning of polymer fibers, 195-212.

Bargteil, A. W., Wojtan, C., Hodgins, J. K., And Turk, G. 2007. A Finite Element Method for Animating Large Viscoplastic Flow. ACM TOG 26, 3 (Jul), 16:1-16:8.

BATTy, C., AND BRIDSOn, R. 2008. Accurate Viscous Free Surfaces for Buckling, Coiling, and Rotating Liquids. In SCA '08, 219-226. 
Bergou, M., Wardetzky, M., Robinson, S., Audoly, B., AND GRINSPUN, E. 2008. Discrete Elastic Rods. ACM TOG 27, 3 (Aug), 63:1-63:12.

Bertails, F., Audoly, B., Cani, M.-P., Querleux, B., Leroy, F., AND LÉvÊQUe, J.-L. 2006. Super-helices for predicting the dynamics of natural hair. ACM TOG 25, 3 (Jul), $1180-1187$.

Bonito, A., Picasso, M., AND LAso, M. 2006. Numerical simulation of 3D viscoelastic flows with free surfaces. J. Comput. Phys. 215, 2, 691-716.

Boyer, F., ANd Primault, D. 2004. Finite element of slender beams in finite transformations: a geometrically exact approach. Int. J. Numer. Methods Eng. 59, 5, 669-702.

Bridson, R., AND MÜLler-FISCher, M. 2007. Fluid Simulation. SIGGRAPH 2007 Course Notes.

Chang, Y., Bao, K., LiU, Y., Zhu, J., And Wu, E. 2009. A particle-based method for viscoelastic fluids animation. VRST '09 (Nov), 111-117.

Chentanez, N., Alterovitz, R., Ritchie, D., Cho, L., Hauser, K. K., GoldberG, K., Shewchuk, J. R., And O'BRIEN, J. F. 2009. Interactive Simulation of Surgical Needle Insertion and Steering. ACM TOG 28, 3 (Jul), 88:1-88:10.

Chiu-Webster, S., ANd Lister, J. R. 2006. The fall of a viscous thread onto a moving surface: a 'fluid-mechanical sewing machine'. J. Fluid Mech. 569, 89-111.

Clavet, S., Beaudoin, P., And Poulin, P. 2005. Particlebased Viscoelastic Fluid Simulation. In SCA '05.

Desbrun, M., And GAscuel, M. 1996. Smoothed particles: A new paradigm for animating highly deformable bodies. Computer Animation and Simulation (Jan), 61-76.

Dewynne, J. N., Ockendon, J. R., And Wilmott, P. 1992. A systematic derivation of the leading-order equations for extensional flows in slender geometries. J. Fluid Mech. 24, 323-338.

DiVerdi, S., Krishnaswamy, A., And Hadap, S. 2010. Industrial-Strength Painting with a Bristle Brush Simulation. submitted.

EgGers, J., AND Dupont, T. F. 1994. Drop formation in a onedimensional approximation of the Navier-Stokes equation. $J$. Fluid Mech. 262, 205-221.

EnTOV, V. M., AND YARIN, A. L. 1984. The dynamics of thin liquid jets in air. J. Fluid Mech. 140, 91-111.

Fedkiw, R., Stam, J., And Jensen, H. W. 2001. Visual Simulation of Smoke. In SIGGRAPH 2001, 15-22.

Foster, N., AND FedKIW, R. 2001. Practical Animation of Liquids. In SIGGRAPH 2001, 23-30.

Foster, N., And Metaxas, D. 1997. Modeling the Motion of a Hot, Turbulent Gas. In SIGGRAPH 97, 181-188.

Gerszewski, D., Bhattacharya, H., And Bargteil, A. W. 2009. A Point-based Method for Animating Elastoplastic Solids. In $S C A$ '09.

Goktekin, T. G., BARgteil, A. W., And O'Brien, J. F. 2004. A method for animating viscoelastic fluids. ACM TOG 23, 3 (Aug), 463-468.
GrÉGoIRE, M., AND SCHÖMER, E. 2007. Interactive simulation of one-dimensional flexible parts. Comput.-Aided Des. 39, 8, 694-707.

Hauth, M., Etzmuss, O., And Strasser, W. 2003. Analysis of numerical methods for the simulation of deformable models. Vis. Comp. 19, 7-8, 581-600.

Hong, J.-M., AND KIM, C.-H. 2005. Discontinuous fluids. ACM TOG 24, 3 (Jul), 915-920.

IRVING, G. 2007. Methods for the physically based simulation of solids and fluids. $\mathrm{PhD}$ thesis, Stanford University.

KAldor, J. M., JAMES, D. L., AND MARschner, S. 2010. Efficient Yarn-based Cloth with Adaptive Contact Linearization. ACM TOG 29, 4 (Jul).

Kharevych, L., Yang, W., Tong, Y., Kanso, E., Marsden, J. E., Schröder, P., And Desbrun, M. 2006. Geometric, Variational Integrators for Computer Animation. In $S C A$ '06, 43-51.

KIM, D., Song, O.-Y., AND Ko, H.-S. 2009. Stretching and Wiggling Liquids. ACM TOG 28, 5 (Dec), 120:1-120:7.

KIRCHHOFF, G. 1859. Über das Gleichgewicht und die Bewegung eines unendlich dünnen elastischen Stabes. Journal für die reine und angewandte Mathematik 56, 285-313.

LANDAU, L. D., AND Lifshitz, E. M. 1981. Theory of Elasticity (Course of Theoretical Physics), $2^{\text {nd }}$ ed. Pergamon Press.

LANGER, J., AND Singer, D. 1996. Lagrangian aspects of the Kirchhoff elastic rod. SIAM Review, 605-618.

Le Merrer, M., Seiwert, J., Quéré, D., and Clanet, C. 2008. Shapes of hanging viscous filaments. EPL 84, 56004.

LeE, S., Olsen, S., AND Gooch, B. 2006. Interactive 3D fluid jet painting. NPAR '06 (Jun), 97-104.

Losasso, F., Gibou, F., And Fedkiw, R. 2004. Simulating water and smoke with an octree data structure. ACM TOG 23, 3 (Aug), 457-462.

Miller, G., And Pearce, A. 1989. Globular dynamics: A connected particle system for animating viscous fluids. COMP. GRAPH. (Jan), 305-309.

Morris, S. W., Dawes, J. H. P., Ribe, N. M., AND Lister, J. R. 2008. Meandering instability of a viscous thread. Phys. Rev. E (Statistical, Nonlinear, and Soft Matter Physics) 77, 6, 066218 .

Müller, M., Charypar, D., And Gross, M. 2003. Particlebased fluid simulation for interactive applications. In SCA '03, 154-159.

Müller, M., Keiser, R., Nealen, A., Pauly, M., Gross, M., AND AleXA, M. 2004. Point based animation of elastic, plastic and melting objects. In SCA '04, 141-151.

Nealen, A., Müller, M., Keiser, R., Boxerman, E., And CARlson, M. 2006. Physically Based Deformable Models in Computer Graphics. CGF 25, 4, 809-836.

O’Brien, J. F., Bargteil, A. W., And Hodgins, J. K. 2002. Graphical Modeling and Animation of Ductile Fracture. ACM TOG 21, 3 (Jul), 291-294.

Oishi, C. M., Tomé, M. F., Cuminato, J. A., And McKee, S. 2008. An implicit technique for solving 3D low Reynolds 
number moving free surface flows. J. Comput. Phys. 227, 16, 7446-7468.

PAI, D. K. 2002. STRANDS: Interactive Simulation of Thin Solids using Cosserat Models. CGF 21, 3, 347-352.

Panda, S., Marheineke, N., And Wegener, R. 2008. Systematic derivation of an asymptotic model for the dynamics of curved viscous fibers. Math. Meth. Appl. Sci. 31, 10, 1153-1173.

RADOVITZKY, R., AND ORTIZ, M. 1999. Error estimation and adaptive meshing in strongly nonlinear dynamic problems. Comput. Methods Appl. Mech. Eng 172, 1-4, 203-240.

Rafiee, A., Manzari, M. T., And Hosseini, M. 2007. An incompressible SPH method for simulation of unsteady viscoelastic free-surface flows. Int. J. Non Linear Mech. 42, 10, 12101223.

Ribe, N. M., Huppert, H. E., Hallworth, M. A., Habibi, M., AND BONN, D. 2006. Multiple coexisting states of liquid rope coiling. J. Fluid Mech. 555, 1, 275-297.

Ribe, N. M. 2004. Coiling of viscous jets. Proc. Math., Phys. and Eng. Sci., 3223-3239.

Skorobogatiy, M., And Mahadevan, L. 2000. Folding of viscous sheets and filaments. EPL 52, 5, 532-538.

Spillmann, J., And Teschner, M. 2007. CORDE: Cosserat Rod Elements for the Dynamic Simulation of One-Dimensional Elastic Objects. In SCA '07, 63-72.

Spillmann, J., And Teschner, M. 2008. An Adaptive Contact Model for the Robust Simulation of Knots. CGF 27, 2, 497-506.

STAM, J. 1999. Stable Fluids. In SIGGRAPH 99, 121-128.

Steele, K., Cline, D., Egbert, P., And Dinerstein, J. 2004. Modeling and rendering viscous liquids. CAVW (Jan), 183-192.

Stora, D., Agliati, P.-O., Cani, M.-P., Neyret, F., And GAscuel, J.-D. 1999. Animating lava flows. GI '99 (Jan), 203-210.

Strutt, J. W. 1945. Theory of Sound, vol. 2. Dover Publications.

TAYLOR, G. I. 1968. Instability of jets, threads, and sheets of viscous fluid. In Proc. 12th Intl Congr. Appl. Mech., Stanford, Springer, Ed., 382.

Terzopoulos, D., And Fleischer, K. 1988. Modeling Inelastic Deformation: Viscoelasticity, Plasticity, Fracture. In SIGGRAPH 88, 269-278.

Terzopoulos, D., Platt, J., And Fleischer, K. 1991. Heating and melting deformable models. J. Visual. Comp. Animat. 2, $2,68-73$.

Theetten, A., Grisoni, L., Duriez, C., And Merlhiot, X. 2007. Quasi-dynamic splines. In SPM '07, ACM, New York, 409-414.

Theetten, A., Grisoni, L., Andriot, C., And Barsky, B. 2008. Geometrically exact dynamic splines. Comput.-Aided Des. $40,1,35-48$.

Trouton, F. R. S. 1906. On the coefficient of viscous traction and its relation to that of viscosity. Proc. Royal Soc. London, A $77,426-440$.

Witkin, A., AND BARAFF, D. 2001. Physically Based Modeling: Principles and Practice. SIGGRAPH 2001 Course Notes.
Wojtan, C., And Turk, G. 2008. Fast Viscoelastic Behavior with Thin Features. ACM TOG 27, 3 (Aug), 47:1-47:8.

\section{A Bending and twisting: gradient \& Hessian}

When using time-parallel directors, bending and twisting energy, gradient, and Hessian are local quantities. It hence suffices to consider three consecutive vertices $\mathbf{x}_{i-1}, \mathbf{x}_{i}$, and $\mathbf{x}_{i+1}$ along the centerline and corresponding consecutive edges $\mathbf{e}^{i-1}$ and $\mathbf{e}^{i}$, with unit tangents $\mathbf{t}^{i-1}$ and $\mathbf{t}^{i}$, respectively. The curvature binormal (associated with the middle vertex $)$ is given by $\kappa \mathbf{b}=2\left(\mathbf{t}^{i-1} \times \mathbf{t}^{i}\right) / \chi$, where $\chi=1+\left(\mathbf{t}^{i-1} \cdot \mathbf{t}^{i}\right)$. Denoting material frames by $\left(\mathbf{d}_{1}^{i-1}, \mathbf{d}_{2}^{i-1}\right)$ and $\left(\mathbf{d}_{1}^{i}, \mathbf{d}_{2}^{i}\right)$, the vertex-based material curvatures take the form

$$
\kappa_{1}=\frac{1}{2}\left(\mathbf{d}_{2}^{i-1}+\mathbf{d}_{2}^{i}\right) \cdot \kappa \mathbf{b} \quad \text { and } \quad \kappa_{2}=-\frac{1}{2}\left(\mathbf{d}_{1}^{i-1}+\mathbf{d}_{1}^{i}\right) \cdot \kappa \mathbf{b} .
$$

Finally, we denote twist by $m$ (associated with the middle vertex), and we abbreviate $\tilde{\mathbf{t}}=\left(\mathbf{t}^{i-1}+\mathbf{t}^{i}\right) / \chi$ and $\tilde{\mathbf{d}_{\alpha}}=\left(\mathbf{d}_{\alpha}^{i-1}+\mathbf{d}_{\alpha}^{i}\right) / \chi$.

Gradients When taking derivatives, the only nontrivial quantities to compute are the derivatives of twist and material curvatures with respect to vertex positions, or equivalently, with respect to variations of edges. The twist gradient is given by

$$
\frac{\partial m}{\partial \mathbf{e}^{i-1}}=\frac{\kappa \mathbf{b}}{2\left|\mathbf{e}^{i-1}\right|} \quad \text { and } \quad \frac{\partial m}{\partial \mathbf{e}^{i}}=\frac{\kappa \mathbf{b}}{2\left|\mathbf{e}^{i}\right|} .
$$

The gradients of material curvatures take the form

$$
\begin{aligned}
\frac{\partial \kappa_{1}}{\partial \mathbf{e}^{i-1}} & =\frac{1}{\left|\mathbf{e}^{i-1}\right|}\left(-\kappa_{1} \tilde{\mathbf{t}}+\mathbf{t}^{i} \times \tilde{\mathbf{d}_{2}}\right), \\
\frac{\partial \kappa_{1}}{\partial \mathbf{e}^{i}} & =\frac{1}{\left|\mathbf{e}^{i}\right|}\left(-\kappa_{1} \tilde{\mathbf{t}}-\mathbf{t}^{i-1} \times \tilde{\mathbf{d}_{2}}\right),
\end{aligned}
$$

and similarly for the gradient of $\kappa_{2}$.

Hessians For the second variation of twist we obtain

$$
\begin{aligned}
\frac{\partial^{2} m}{\partial\left(\mathbf{e}^{i-1}\right)^{2}} & =-\frac{1}{4\left|\mathbf{e}^{i-1}\right|^{2}}\left(\kappa \mathbf{b} \otimes\left(\mathbf{t}^{i-1}+\tilde{\mathbf{t}}\right)+\left(\mathbf{t}^{i-1}+\tilde{\mathbf{t}}\right) \otimes \kappa \mathbf{b}\right), \\
\frac{\partial^{2} m}{\partial\left(\mathbf{e}^{i}\right)^{2}} & =-\frac{1}{4\left|\mathbf{e}^{i}\right|^{2}}\left(\kappa \mathbf{b} \otimes\left(\mathbf{t}^{i}+\tilde{\mathbf{t}}\right)+\left(\mathbf{t}^{i}+\tilde{\mathbf{t}}\right) \otimes \kappa \mathbf{b}\right), \\
\frac{\partial^{2} m}{\partial \mathbf{e}^{i-1} \partial \mathbf{e}^{i}} & =\left(\frac{\partial^{2} m}{\partial \mathbf{e}^{i} \partial \mathbf{e}^{i-1}}\right)^{T}=\frac{1}{2\left|\mathbf{e}^{i-1}\right|\left|\mathbf{e}^{i}\right|}\left(\frac{2}{\chi}\left[\mathbf{t}^{i-1}\right]_{\times}-\kappa \mathbf{b} \otimes \tilde{\mathbf{t}}\right) .
\end{aligned}
$$

Here, $[\mathbf{t}]_{\times}$denotes the $3 \times 3$ skew-symmetric matrix acting on 3 -vectors $\mathbf{x}$ by $[\mathbf{t}]_{\times} \mathbf{x}=\mathbf{t} \times \mathbf{x}$, and $\partial^{2} m / \partial \mathbf{e}^{i-1} \partial \mathbf{e}^{i}$ denotes taking the derivative first with respect to $\mathbf{e}^{i-1}$ and then with respect to $\mathbf{e}^{i}$. The second variations of material curvatures are given by

$$
\begin{aligned}
& \frac{\partial^{2} \kappa_{1}}{\partial\left(\mathbf{e}^{i-1}\right)^{2}}=\frac{1}{\left|\mathbf{e}^{i-1}\right|^{2}}\left(2 \kappa_{1} \tilde{\mathbf{t}} \otimes \tilde{\mathbf{t}}-\left(\mathbf{t}^{i} \times \tilde{\mathbf{d}_{2}}\right) \otimes \tilde{\mathbf{t}}-\tilde{\mathbf{t}} \otimes\left(\mathbf{t}^{i} \times \tilde{\mathbf{d}_{2}}\right)\right) \\
& -\frac{1}{\chi\left|\mathbf{e}^{i-1}\right|^{2}} \kappa_{1}\left(\mathrm{I}_{3}-\mathbf{t}^{i-1} \otimes \mathbf{t}^{i-1}\right) \\
& +\frac{1}{4\left|\mathbf{e}^{i-1}\right|^{2}}\left(\kappa \mathbf{b} \otimes \mathbf{d}_{2}^{i-1}+\mathbf{d}_{2}^{i-1} \otimes \kappa \mathbf{b}\right), \\
& \frac{\partial^{2} \kappa_{1}}{\partial\left(\mathbf{e}^{i}\right)^{2}}=\frac{1}{\left|\mathbf{e}^{i}\right|^{2}}\left(2 \kappa_{1} \tilde{\mathbf{t}} \otimes \tilde{\mathbf{t}}+\left(\mathbf{t}^{i-1} \times \tilde{\mathbf{d}_{2}}\right) \otimes \tilde{\mathbf{t}}+\tilde{\mathbf{t}} \otimes\left(\mathbf{t}^{i-1} \times \tilde{\mathbf{d}_{2}}\right)\right) \\
& -\frac{1}{\chi\left|\mathbf{e}^{i}\right|^{2}} \kappa_{1}\left(\mathrm{I}_{3}-\mathbf{t}^{i} \otimes \mathbf{t}^{i}\right) \\
& +\frac{1}{4\left|\mathbf{e}^{i}\right|^{2}}\left(\kappa \mathbf{b} \otimes \mathbf{d}_{2}^{i}+\mathbf{d}_{2}^{i} \otimes \kappa \mathbf{b}\right), \\
& \frac{\partial^{2} \kappa_{1}}{\partial \mathbf{e}^{i-1} \partial \mathbf{e}^{i}}=\left(\frac{\partial^{2} \kappa_{1}}{\partial \mathbf{e}^{i} \partial \mathbf{e}^{i-1}}\right)^{T}=\frac{-\kappa_{1}}{\chi\left|\mathbf{e}^{i-1}\right|\left|\mathbf{e}^{i}\right|}\left(\mathrm{I}_{3}+\mathbf{t}^{i-1} \otimes \mathbf{t}^{i}\right) \\
& +\frac{2 \kappa_{1} \tilde{\mathbf{t}} \otimes \tilde{\mathbf{t}}-\left(\mathbf{t}^{i} \times \tilde{\mathbf{d}_{2}}\right) \otimes \tilde{\mathbf{t}}+\tilde{\mathbf{t}} \otimes\left(\mathbf{t}^{i-1} \times \tilde{\mathbf{d}_{2}}\right)-\left[\tilde{\mathbf{d}}_{2}\right]_{\times}}{\left|\mathbf{e}^{i-1}\right|\left|\mathbf{e}^{i}\right|}
\end{aligned}
$$

and similarly for $\kappa_{2}$. 